\title{
Subgrouping of Fibromyalgia Patients on the Basis of Pressure-Pain Thresholds and Psychological Factors
}

\author{
Thorsten Giesecke, ${ }^{1}$ David A. Williams, ${ }^{1}$ Richard E. Harris,${ }^{1}$ Thomas R. Cupps, ${ }^{2}$ \\ Xiaoming Tian, ${ }^{3}$ Thomas X. Tian, ${ }^{3}$ Richard H. Gracely, ${ }^{1}$ and Daniel J. Clauw ${ }^{1}$
}

Objective. Although the American College of Rheumatology (ACR) criteria for fibromyalgia are used to identify individuals with both widespread pain and tenderness, individuals who meet these criteria are not a homogeneous group. Patients differ in their accompanying clinical symptoms, as well as in the relative contributions of biologic, psychological, and cognitive factors to their symptom expression. Therefore, it seems useful to identify subsets of fibromyalgia patients on the basis of which of these factors are present. Previous attempts at identifying subsets have been based solely on psychological and cognitive features. In this study, we attempt to identify patient subsets by incorporating these features as well as the degree of hyperalgesia/ tenderness, which is a key neurobiologic feature of this illness.

Methods. Ninety-seven individuals meeting the ACR criteria for fibromyalgia finished the same battery of self-report and evoked-pain testing. Analyzed variables were obtained from several domains, consisting of 1) mood (evaluated by the Center for Epidemiologic Studies Depression Scale [for depression] and the State-Trait Personality Inventory [for symptoms of trait-related anxiety]), 2) cognition (by the catastrophizing and control of pain subscales of the Coping

Supported by Department of Army grant DAMD 17-00-20018, NIH grant 5-R01-AT 000004-02, and grant 5-M01-RR13297 from the General Clinical Research Center Program of the National Center for Research Resources, NIH.

${ }^{1}$ Thorsten Giesecke, MD, David A. Williams, PhD, Richard E. Harris, PhD, Richard H. Gracely, PhD, Daniel J. Clauw, MD: University of Michigan, Ann Arbor; ${ }^{2}$ Thomas R. Cupps, MD: Georgetown University, Washington, DC; ${ }^{3}$ Xiaoming Tian, MD, Thomas X. Tian, MD: Academy of Acupuncture and Chinese Medicine, Bethesda, Maryland.

Address correspondence and reprint requests to Daniel $\mathrm{J}$. Clauw, MD, University of Michigan Health System, Department of Internal Medicine, Division of Rheumatology, 300 NIB NI7B10, Ann Arbor, MI 48109-0483. E-mail: dclauw@med.umich.edu.

Submitted for publication February 12, 2003; accepted in revised form June 6, 2003
Strategies Questionnaire), and 3) hyperalgesia/ tenderness (by dolorimetry and random pressure-pain applied at suprathreshold values). Cluster analytic procedures were used to distinguish subgroups of fibromyalgia patients based on these domains.

Results. Three clusters best fit the data. Multivariate analysis of variance (ANOVA) confirmed that each variable was differentiated by the cluster solution (Wilks' $\lambda$ [degrees of freedom 6,89] $=0.123, P<$ 0.0001), with univariate ANOVAs also indicating significant differences (all $P<\mathbf{0 . 0 5}$ ). One subgroup of patients $(n=50)$ was characterized by moderate mood ratings, moderate levels of catastrophizing and perceived control over pain, and low levels of tenderness. A second subgroup $(n=31)$ displayed significantly elevated values on the mood assessments, the highest values on the catastrophizing subscale, the lowest values for perceived control over pain, and high levels of tenderness. The third group $(n=16)$ had normal mood ratings, very low levels of catastrophizing, and the highest level of perceived control over pain, but these subjects showed extreme tenderness on evoked-pain testing.

Conclusion. These data help support the clinical impression that there are distinct subgroups of patients with fibromyalgia. There appears to be a group of fibromyalgia patients who exhibit extreme tenderness but lack any associated psychological/cognitive factors, an intermediate group who display moderate tenderness and have normal mood, and a group in whom mood and cognitive factors may be significantly influencing the symptom report.

Fibromyalgia (FM) is a complex, contentious disease entity. It is currently defined by the 1990 American College of Rheumatology (ACR) classification criteria (1), in which an individual is required to have both a history of chronic widespread pain and the finding of 
11 of 18 possible tender points on examination. Tender points are located in 9 paired regions of the body, and if an individual reports pain when one of these regions is palpated with 4 kilograms of pressure, this is considered a positive tender point. Using the ACR criteria, the prevalence of FM in industrialized countries ranges from $0.5 \%$ to $4 \%$ (2).

There is, however, tremendous variability within individuals whose disease manifestations match the ACR criteria. Some individuals with FM experience only pain, while others experience a variety of additional symptoms. Moreover, there is substantial overlap between FM and other systemic entities (e.g., chronic fatigue syndrome) and regional syndromes (e.g., irritable bowel syndrome or temporomandibular disorders) (3). Factor analytic techniques have been used in population-based studies to identify the seminal features of these conditions; in such studies, the key symptoms that coaggregate are multifocal pain, fatigue, memory difficulties, and mood disturbances (4). The term chronic multisymptom illness has been coined by investigators from the United States Centers for Disease Control and Prevention to describe this symptom complex (5).

Given this heterogeneity of symptoms, it has become increasingly clear that FM is not a discrete entity, but rather one of many overlapping syndromes and symptoms. Because of this, many investigators in the field have suggested that there is a need for empirically derived subgroups, ideally using constructs that would help tailor more specific therapies to each patient (6-8). Several studies have used sophisticated, multivariate cluster analytic techniques to identify replicable, homogeneous subgroups of patients with chronic pain within heterogeneous chronic pain populations. Bradley et al (9) applied these methods to the analysis of personality profiles in patients with chronic low back pain. Cluster analytic methods revealed that there were distinct subgroups of patients with chronic pain, distinguished on the basis of their Minnesota Multiphasic Personality Inventory profiles. Keefe et al (10) used similar methods to identify subgroups of patients with low back pain who differed in terms of their overt pain behavior. Distinct subgroups of patients divided on the basis of pain beliefs as well as subgroups distinguished on the basis of patients' responses to the Symptom Checklist 90 revised have also been identified using cluster analytic methods $(11,12)$.

Turk et al (8) were the first investigators to show that subgroups identified by cluster analysis, based upon the Multidimensional Pain Inventory in various chronic pain populations, could also be applicable to a population of FM patients, and that each of these FM subgroups responds differently to treatment (13). In this subgrouping performed by Turk et al among patients with FM, psychosocial and cognitive characteristics of the patients were used to distinguish the groups. In addition to psychosocial and cognitive factors, however, increasing evidence suggests a strong neurobiologic factor. Much of this work has been focused on determining the mechanisms that might be operative in the hyperalgesia (increased sensitivity to painful stimuli) and allodynia (sensitivity to normally nonpainful stimuli) seen in this condition. Thus, it would be useful if a measure of hyperalgesia could be incorporated into patient subgroupings.

One reason that hyperalgesia has not been used in the past is that the measure of tenderness most often used to assess FM, the tender point count, has many features that confound its use in these types of models. One problem with the tender point count is statistical; within a group of FM patients, the most frequent results will be 17 or 18 tender points. This abnormal distribution and narrow range make it difficult to differentiate patients using this measure. Another problem with the use of tender points is that it is highly correlated with measures of psychological distress $(14,15)$. This is problematic in examining the independent contributions that pressure-pain sensitivity and distress may make to tenderness in subgroups of patients. Recent work has made it clear that other measures of pressure-pain sensitivity, especially those in which stimuli are presented in a random manner such that individuals cannot anticipate the next stimulus, have a much wider range, have a normal distribution, and are not correlated with measures of distress (15).

Thus, in the present study, we identified a group of patients with FM on the basis of the ACR classification criteria, and we examined the manner in which neurobiologic, psychological, and cognitive features could be clustered to form subgroups of FM patients.

\section{PATIENTS AND METHODS}

Patients. One hundred seventeen patients (102 female and 15 male, ages 18-60 years) who met the 1990 ACR criteria for the classification of FM (1) were recruited. Seven of these patients (6 female, 1 male) were referrals to a tertiary rheumatology outpatient clinic, and 110 were recruited by newspaper advertisements. The patients were studied at the General Clinical Research Center of Georgetown University Medical Center. All patients underwent a comprehensive screening during which the diagnosis was confirmed and comorbidities 
were evaluated. Exclusion criteria were severe physical impairment (e.g., bilateral amputation, complete blindness, or deafness), medical conditions that were capable of causing patients symptoms (e.g., morbid obesity, autoimmune/inflammatory diseases), cardiopulmonary disorders (i.e., angina, congestive heart failure, chronic obstructive pulmonary disease, or chronic asthma), uncontrolled endocrine or allergic disorders (i.e., hyper-/hypothyroidism, diabetes, or allergic rhinitis), malignancy, severe psychiatric illnesses (e.g., current schizophrenia, substance abuse within 2 years), factors known to affect the hypothalamic-pituitary-adrenal axis or autonomic function (e.g., cigarette smoking, daily intake of caffeine exceeding the equivalent of 2 cups of coffee), and usage of medications other than as-needed analgesics (excluding long-term use of narcotics) and appropriate dosages of thyroid hormone.

When subjects were considered qualified for inclusion in the study, they were given an appointment to begin participation in a 2-day study protocol. They were asked to discontinue intake of antidepressants within 1 week prior to the appointment, but were allowed to take nonsteroidal antiinflammatory drugs until 3 days before the appointment. On the first day of the study, patients completed the self-report questionnaires and were familiarized with the pain testing. On the following day, pain testing was performed.

Self-report measures. Visual analog scale (VAS). Clinical pain was reported on a $0-100$ VAS, with 0 being "no pain" and 100 being "the worst pain imaginable."

Center for Epidemiologic Studies Depression Scale (CES-D). The CES-D (16) is a 20-item self-report questionnaire that assesses symptoms of depression in nonpsychiatric adults. This instrument possesses strong psychometric properties and has demonstrated strong associations with other measures of depressive symptoms (17). Cut-off scores have been established to specify that scores in excess of 16 points are indicative of "probable depression."

State-Trait Personality Inventory (STPI). Symptoms of trait anxiety were assessed using the STPI (Form Y) (18). The STPI is an 80-item self-report questionnaire with eight 10 -item scales for measuring state-related and trait-related anxiety, anger, depression, and curiosity. For purposes of this study, the 10 -item scale measuring trait anxiety was used. The STPI possesses strong psychometric properties for the assessment of these mood symptoms, since the items have been well validated as part of larger instruments such as the State-Trait Anxiety Inventory and the State-Trait Anger Inventory (19). Although cut-off values that relate to clinical diagnostic categories have not been established, a score above 22 points on the trait anxiety scale corresponds to the 75 th percentile of the score among normal adults.

Coping Strategies Questionnaire (CSQ). The CSQ was developed by Rosenstiel and Keefe as a measure of the strategies utilized by patients to cope with chronic pain, and as a measure of the perceived efficacy of those strategies when used to control and decrease pain (20). The CSQ assesses the use of 6 cognitive coping strategies (diverting attention, reinterpreting pain sensations, coping self-statements, ignoring pain sensations, praying or hoping, and catastrophizing) and 1 behavioral coping strategy (increasing behavioral activity). Each strategy subscale is composed of 6 items, and subjects are asked to rate the frequency with which they use each strategy on a 7 -point scale $(0=$ never, $3=$ sometimes, $6=$ always $)$. Two single-item scales were used to assess the patients' belief in the effectiveness of the coping strategies used to control or decrease their pain; each rating of efficacy is made on a 7-point scale, with 0 indicating no efficacy and 7 indicating complete efficacy. For the purposes of this study, we used the "catastrophizing" and "control over pain" subscales.

Medical Outcomes Study 36-item Short Form (SF-36) health survey. The SF-36 is a self-report inventory that assesses health across 6 functional domains and has been widely used in outcome evaluation studies (21). In an analysis of the utility of this instrument compared with other scales, this tool has been shown to perform well as a measure of health status (22). For the purposes of this study, the SF-36 physical component summary score was used as a measure of clinical status. Raw scores were transformed to a $0-100$-point scale, with higher scores being indicative of better functioning.

Experimental pain assessments. During the pain testing session, a manual tender point count was performed, followed by a dolorimeter examination in 3 paired points of the body (epicondyle, mid-trapezius, and thumbnail), using a continuous ascending paradigm. Pressure was increased at a rate of $1 \mathrm{~kg} / \mathrm{second}$, and subjects indicated when the stimulus first became painful (pain threshold). The final pain threshold value was the statistical mean of the values of all 6 points. We recently showed that this model predicts overall tenderness with excellent accuracy (23).

Pressure-pain sensitivity was evaluated by subjective scaling of multiple pressure-pain sensations of suprathreshold intensities. Discrete 5-second pressure stimuli were applied to the fixated left thumbnail with a $1-\mathrm{cm}^{2}$ hard rubber probe. Previous studies have shown that "neutral" regions such as the thumb accurately reflect an individual's overall pressure-pain sensitivity (15). The rubber probe was attached to a hydraulic piston, a combination of valves (to control stimulus duration), and a scale. Calibrated weights put on the scale produced controlled, repeatable pressure-pain stimuli of rectangular waveform at the thumbnail. Subjects rated the intensity of pressure-pain sensations using a combined numeric analog descriptor scale, which was developed from previously quantified verbal descriptors (24). First, a series of stimuli was presented in a predictable, "ascending" manner, beginning at $0.5 \mathrm{~kg} / \mathrm{cm}^{2}$ and increasing in $0.5-\mathrm{kg} / \mathrm{cm}^{2}$ intervals up to tolerance or to a maximum of $10 \mathrm{~kg} / \mathrm{cm}^{2}$. Following the ascending series, 36 stimuli were delivered at 20 -second intervals in random order, using the multiple random-staircase (MRS) pressure-pain sensitivity method (25). The MRS method is response dependent, i.e., it determines the stimulus intensity needed to elicit a specified response. For this analysis, we used the stimulus intensities needed to elicit slightly intense pain (13.5/20 pain scale units).

Statistical analysis. Patient subgroups were formed using agglomerative hierarchic cluster analysis, with squared Euclidean distances incorporated in the proximities matrix. Ward's method was used to form clusters at each stage so that the squared within-group deviations around the mean value for each cluster were minimized. This cluster analytic method has been shown to produce the best recovery of structure for clusters of all sizes (26). Following the formation of clusters, multiple analyses of variance (ANOVAs) were used to confirm that each variable used in the analysis was differentiated by the 
Table 1. Patient characteristics and variables used for clustering of the whole study population $(\mathrm{n}=97 ; 85 \text { female, } 12 \text { male })^{*}$

\begin{tabular}{lccc}
\hline \multicolumn{1}{c}{ Variable } & $\begin{array}{c}\text { Maximum } \\
\text { possible range }\end{array}$ & Mean & SD \\
\hline Age, years & $18-60$ & 44.94 & 10.37 \\
No. of tender points & $11-18$ & $14 \dagger$ & - \\
Biologic pressure-pain $¥$ & & & \\
$\quad$ MRS, kg & $0-10$ & 5.12 & 2.29 \\
$\quad$ Dolorimeter, kg & $0-10$ & 4.81 & 2.16 \\
Psychosocial/cognitive & $0-60$ & 17.23 & 9.39 \\
$\quad$ CES-D & $20-80$ & 22.43 & 8.19 \\
$\quad$ STPI-trait anxiety & $0-36$ & 10.81 & 7.30 \\
Catastrophizing on CSQ & $0-6$ & 3.08 & 1.29 \\
$\quad$ Control over pain on CSQ & $0-100$ & 36.55 & 9.28 \\
SF-36 physical functioning & &
\end{tabular}

* MRS = multiple random-staircase pressure-pain sensitivity determination; CES-D = Center for Epidemiologic Studies Depression questionnaire (scores $>16$ indicate probable depression); STPI $=$ StaitTrait Personality Inventory (scores $>22$ on trait anxiety correspond to the 75th percentile for normal adults); CSQ = Coping Strategies Questionnaire (high catastrophizing scores indicate that catastrophizing is an important element of an individual's means of coping, while higher control over pain scores indicates that the individual is more able to use coping strategies to control pain); SF-36 = Short Form 36 (higher scores indicate better functioning).

$\dagger$ Tender point counts are not normally distributed, and therefore the median is displayed, without SD (geometric mean).

$\ddagger$ Lower pain threshold values on MRS and dolorimetry are equivalent to higher tenderness.

cluster solution. All analyses were performed using SPSS for Windows, version 10 (SPSS, Chicago, IL).

\section{RESULTS}

Characteristics of patients. All screened patients finished the study protocol; however, complete data were available on only 97 of the 117 patients. Because missing data distort the results of clustering procedures, we excluded 17 women and 3 men from further analysis. Thus, 85 women $(87.6 \%)$ and 12 men $(12.4 \%)$ were studied in the cluster analysis. The subjects were fairly representative of the US population, with 78 whites (80\%), 11 African Americans (11\%), 3 Asians (3\%), 2 Hispanics (2\%), and 3 subjects of other ethnicity (3\%). The characteristics of the study sample are shown in Table 1.

Clustering and subgrouping. Three clusters best fit the data. The symptom profiles of the 3 clusters are displayed in Figure 1, and corresponding values for the neurobiologic (tenderness on evoked-pain testing), psychosocial, and cognitive variables are displayed in Table 2. Multivariate ANOVAs confirmed that each variable was differentiated by the cluster solution (Wilks' $\lambda$ [degrees of freedom 6,89] $=0.123, P<$ 0.0001). Furthermore, univariate ANOVAs confirmed

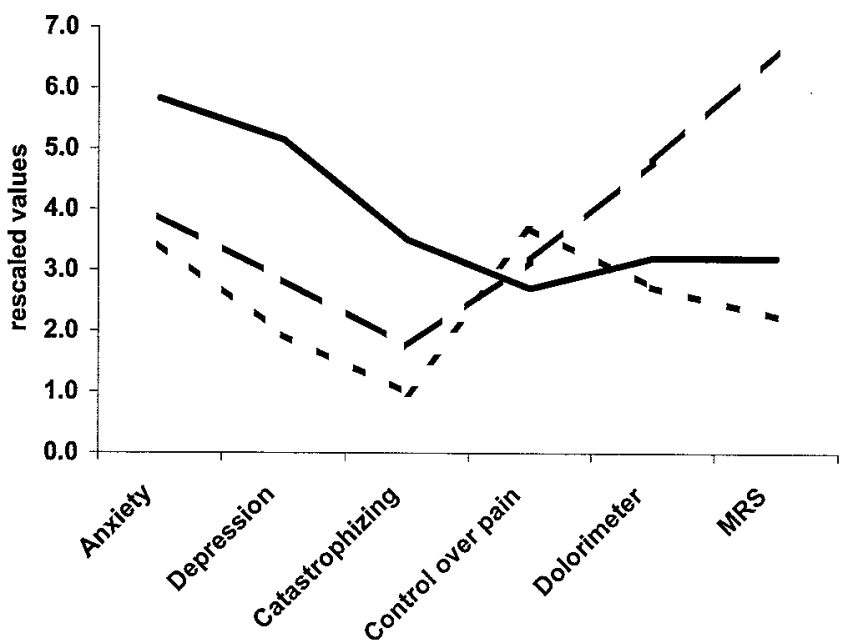

Figure 1. Symptom profiles of patient subgroups, as detected by cluster analysis. Cluster $1(\mathrm{n}=50$; long broken line $)$ displayed moderate levels of anxiety, depression, and catastrophizing, moderate control over pain, as well as moderate to low tenderness. Cluster 2 ( $\mathrm{n}=31$; solid line) was characterized by high levels of anxiety, depression, and catastrophizing, the lowest control over pain, and considerable tenderness. Cluster $3(\mathrm{n}=16$; short broken line) displayed the lowest levels of anxiety, depression, and catastrophizing and the highest control over pain, but also the lowest pain thresholds. Values for anxiety, depression, and catastrophizing have been rescaled (divided by 5 ) to facilitate meaningful simultaneous display. MRS = multiple random-staircase pressure-pain sensitivity determination.

that each variable in the analysis significantly differentiated the clusters (all $P<0.05$ ).

As shown in Figure 2, cluster 1 contained the highest number of patients $(\mathrm{n}=50)$, with 41 women $(82 \%)$ and 9 men $(18 \%)$. This cluster consisted of 39 whites (78\%), 7 African Americans (14\%), 1 Asian

Table 2. Cluster characteristics*

\begin{tabular}{lrrr}
\hline \multicolumn{1}{c}{ Variable } & \multicolumn{1}{c}{ Cluster 1 } & \multicolumn{1}{c}{ Cluster 2 } & \multicolumn{1}{c}{ Cluster 3 } \\
\hline Age, years & $46.1 \pm 10.0$ & $42.8 \pm 11.3$ & $45.5 \pm 9.6$ \\
MRS, kg & $6.63 \pm 1.7$ & $3.22 \pm 1.4$ & $2.24 \pm 1.3$ \\
Dolorimeter, kg & $4.81 \pm 2.2$ & $3.21 \pm 1.7$ & $2.73 \pm 1.3$ \\
CES-D score & $14.16 \pm 7.5$ & $25.74 \pm 7.3$ & $9.63 \pm 4.6$ \\
STPI-trait anxiety & $19.36 \pm 4.9$ & $29.13 \pm 8.5$ & $17.06 \pm 6.3$ \\
$\quad$ score & & & \\
Catastrophizing score & $8.76 \pm 6.1$ & $17.52 \pm 6.0$ & $4.86 \pm 3.0$ \\
Control over pain & $3.16 \pm 1.3$ & $2.71 \pm 1.2$ & $3.69 \pm 1.1$ \\
$\quad$ score & & & \\
SF-36 physical & $37.44 \pm 9.2$ & $35.74 \pm 7.7$ & $34.56 \pm 10.2$ \\
$\quad$ functioning score & & & \\
\hline
\end{tabular}

* Values are the mean \pm SD. Multivariate analysis of variance confirmed that each variable was differentiated by the cluster solution (Wilks' $\lambda$ [degrees of freedom 6,89$]=0.123, P<0.0001$ ), and univariate analysis of variance confirmed that each variable significantly differentiated the clusters (all $P<0.05$ ). See Table 1 for definitions. 


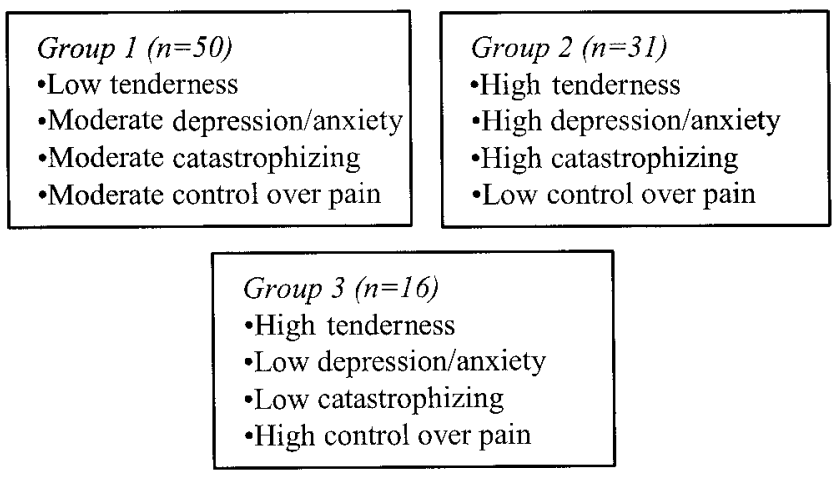

Figure 2. Subgroups of fibromyalgia patients, based on a psychosocial domain (depression/anxiety), a cognitive domain (catastrophizing/ control over pain), and a neurobiologic domain (tenderness).

(2\%), 1 Hispanic (2\%), and 2 of other ethnicity (4\%). These patients were characterized by moderate anxiety on the STPI trait anxiety questionnaire (mean \pm SD score $19.4 \pm 4.9$ ), moderate levels of depression on the CES-D (mean \pm SD score $14.2 \pm 7.5$ ), moderate levels of catastrophizing and control over pain on the CSQ (mean \pm SD scores $8.8 \pm 6.1$ and $3.2 \pm 1.3$, respectively), as well as the highest pain threshold by dolorimetry $(4.8 \pm 2.2 \mathrm{~kg})$ and the lowest suprathreshold painsensitivity level by the MRS method $(6.6 \pm 1.7 \mathrm{~kg})$, i.e., a low level of tenderness. This mean value for pain threshold is plausible because a positive tender point count is not equivalent to a pressure-pain threshold of 4 $\mathrm{kg}$.

Cluster 2 comprised 31 patients, of which 28 were female $(90.3 \%)$ and 3 were male $(9.7 \%)$. This cluster consisted of 24 whites (77\%), 4 African Americans (13\%), 2 Asians (6\%), and 1 Hispanic (3\%). These patients were characterized by the highest levels of anxiety and depressive symptoms (mean \pm SD scores $29.1 \pm 8.5$ and $25.7 \pm 7.3$, respectively) and the highest level of catastrophizing (mean \pm SD score $17.5 \pm 6.0$ )

Table 3. Discriminative analysis of cluster characteristics*

\begin{tabular}{lrrr}
\hline \multicolumn{1}{c}{ Variable } & \multicolumn{1}{c}{ Cluster 1 } & \multicolumn{1}{c}{ Cluster 2 } & \multicolumn{1}{c}{ Cluster 3 } \\
\hline Constant & -18.19352 & -22.53864 & -10.12578 \\
MRS & $\mathbf{2 . 1 7 5 6 2}$ & 0.06690 & 0.26735 \\
Dolorimeter & $\mathbf{1 . 1 6 5 1 9}$ & 0.64933 & 0.47685 \\
CES-D & 0.07713 & $\mathbf{0 . 3 0 4 7 4}$ & 0.07287 \\
STPI-trait anxiety & 0.36064 & $\mathbf{0 . 5 9 6 2 5}$ & 0.37779 \\
Catastrophizing & 0.24672 & $\mathbf{0 . 6 1 2 2 9}$ & 0.27448 \\
Control over pain & 1.93189 & 2.52485 & $\mathbf{2 . 6 7 5 0 5}$ \\
\hline
\end{tabular}

* Values are the coefficients for discriminant functions for each cluster (relative to the constants) (see text for details on assignment of patients to clusters). Values in boldface indicate that the variable loads highest for that particular cluster. See Table 1 for definitions. combined with the lowest levels of control over pain (mean \pm SD score $2.7 \pm 1.2$ ). They also displayed considerable tenderness, with a mean \pm SD pain threshold of $3.2 \pm 1.7 \mathrm{~kg}$ and a suprathreshold pain-sensitivity level of $3.2 \pm 1.4 \mathrm{~kg}$.

Cluster 3 was the smallest cluster $(n=16)$ and consisted solely of women. Fifteen women were white $(94 \%)$ and 1 was of other ethnicity (6\%). These patients were characterized by the lowest levels of anxiety (mean \pm SD score $17.1 \pm 6.3$ ), depressive symptoms (mean \pm SD score 9.6 \pm 4.6), and catastrophizing (mean \pm SD score $4.9 \pm 3.0$ ) and had the highest level of control over pain (mean \pm SD score $3.7 \pm 1.1$ ). Nevertheless, they had, by far, the lowest pain threshold and the highest suprathreshold pain-sensitivity level (mean $\pm \mathrm{SD} 2.7 \pm 1.3 \mathrm{~kg}$ and $2.2 \pm 1.3 \mathrm{~kg}$, respectively), i.e., the highest level of tenderness.

Tender points, pain, and physical functioning among subgroups. The mean \pm SD tender point counts in clusters 1,2 , and 3 were $13 \pm 3,13 \pm 4$, and $16 \pm 3$, respectively. Self-reported mean \pm SD pain scores on a regular 0-100 VAS in clusters 1,2 , and 3 were $52 \pm 26$, $53 \pm 27$, and $62 \pm 20$, respectively. Values for perceived physical functioning, as determined by the SF-36 for clusters 1,2, and 3, are displayed in Table 2. There were no significant differences between the clusters with regard to the number of tender points, extent of selfreported pain, and perceived level of physical functioning.

To facilitate the task of assigning individual patients to our clusters, we performed a discriminative analysis (results shown in Table 3). A constant and a set of 6 coefficients for the 6 variables are given for each cluster. After multiplying the coefficient and the actual value of each variable, the sum of the constant and these 6 products is calculated. Application of the 3 formulas provides 3 values for every patient. The highest value determines the cluster to which the patient is assigned.

\section{DISCUSSION}

Our study showed that within a group composed entirely of individuals with FM, there are subgroups of patients who can be identified on the basis of their patterns of pressure-pain sensitivity, mood, and cognition. Over half of the patients in our sample were in cluster 1, in which patients had moderately increased pressure-pain sensitivity and mood ratings and average cognitive factors. This might represent the "typical" FM patient, especially those seen in a primary care setting. The next largest group had highly elevated pressure-pain sensitivity and had significantly high values in terms of 
the presence of mood and cognitive factors. This might characterize the presentation of FM common in tertiary care settings. The smallest group, cluster 3 , had extremely elevated pressure-pain sensitivity, but had no identifiable psychological or cognitive factors that appeared to be contributing to increased symptom reporting. This group may characterize the neurobiologic presentation of FM when it is unconfounded by common psychological factors. In summary, these results add to existing data and further the clinical impression that FM is a heterogeneous disorder.

With regard to the measures of tender points (tender point counts), physical functioning (SF-36), and self-reported pain, a comparison of these 3 measures among the 3 clusters showed that none was associated with subgroup membership. Studies comparing the tender point count with other measures of tenderness have found that the tender point count is significantly influenced by various measures of psychological distress $(2,14,15)$. Petzke et al also found a relationship between the tender point count and self-reported pain (15). In contrast, in accordance with other results from this study, tender point counts in our 3 subgroups were not significantly associated with the other, "purer" measures of pressure-pain sensitivity. The tender point count may serve as a tool to attain an overall evaluation of physiologic and psychological dysfunction, but as a compound measure of many factors, it does not reflect differences in distress or pressure-pain sensitivity or provide help in subgrouping FM patients.

The similarity of self-reported pain among the 3 subgroups demonstrates once more that the relationship between pain and depression is still very unclear. Although the severity of pain has been shown to be associated with the severity of depression in FM patients (27), the cross-sectional relationship of pain and depression may become nonsignificant once other diseaserelated variables are controlled (28).

This study also sheds new light on the relationship between pressure-pain sensitivity and distress. Distress is typically operationalized as some combination of somatic symptoms and symptoms of anxiety/depression. Until recently it had been assumed that because tender point counts measure pressure-pain sensitivity, and because the presence of tender points is associated with distress, an individual's sensitivity to mechanical pressure was associated with distress $(29,30)$. However, as already pointed out, in a study of evoked-pain measures by Petzke et al (15), this is not necessarily the case. Tender points are a flawed measure of "pure" pressurepain sensitivity, because, in large part, they are influ- enced considerably by distress. More sophisticated measures of pressure-pain sensitivity appear to be independent of psychological status. Dolorimetry results are only mildly influenced by distress, thus being a purer measure than is the tender point count, but even dolorimetry is not as independent of distress as are the more sophisticated measures $(14,15)$. As a result, in this study, we see 2 groups of patients with high levels of pressurepain sensitivity, of which one displayed high levels of distress (cluster 2) and the other displayed very low levels of distress (cluster 3 ).

The implications of these results with regard to treatment of FM are not yet clear. It might be expected that cluster 1 , in which patients had moderate mood difficulties and already had very adaptive cognitive factors, might be less likely to need or respond to cognitive behavioral approaches, whereas cluster 2 , in which patients had extremely high levels of distress, might benefit most from this type of program. Pharmacologic therapy using antidepressant drugs, selective serotonin reuptake inhibitors, and other psychotropic medications might also be expected to be more effective in cluster 2, since mood appeared to play a greater role in driving symptom expression. However, cluster 3 may possibly respond best to symptom-based pharmacologic therapy, including antidepressant agents with analgesic properties. Further studies need to replicate these findings, and additional testing should be done to determine whether these FM subgroupings can, in fact, be used to identify optimal treatment strategies.

\section{REFERENCES}

1. Wolfe F, Smythe HA, Yunus MB, Bennett RM, Bombardier C, Goldenberg DL, et al. The American College of Rheumatology 1990 criteria for the classification of fibromyalgia: report of the multicenter criteria committee. Arthritis Rheum 1990;33:160-72.

2. Wolfe F, Ross K, Anderson J, Russell IJ. Aspects of fibromyalgia in the general population: sex, pain threshold, and fibromyalgia symptoms. J Rheumatol 1995;22:151-6.

3. Aaron LA, Buchwald D. A review of the evidence for overlap among unexplained clinical conditions. Ann Intern Med 2001;134: 868-81.

4. Doebbeling BN, Clarke WR, Watson D, Torner JC, Woolson RF, Voelker MD, et al. Is there a Persian Gulf War syndrome? Evidence from a large population-based survey of veterans and nondeployed controls. Am J Med 2000;108:695-704.

5. Fukuda K, Nisenbaum R, Stewart G, Thompson WW, Robin L, Washko RM, et al. Chronic multisymptom illness affecting Air Force veterans of the Gulf War. JAMA 1998;280:981-8.

6. Bennett RM, Campbell S, Burckhardt C, Clark S, O'Reilly C, Wiens A. A multidisciplinary approach to fibromyalgia management: balanced approach provides small but significant gains. J Musculoskeletal Medicine 1991;8:21-32.

7. Masi AT, Yunus MB. Fibromyalgia: which is the best treatment? A personalized, comprehensive, ambulatory, patient-involved 
management programme. Baillieres Clin Rheumatol 1991;4: 333-70.

8. Turk DC, Okifuji A, Sinclair JD, Starz TW. Pain, disability, and physical functioning in subgroups of patients with fibromyalgia. J Rheumatol 1996;23:1255-62.

9. Bradley LA, Prokop CK, Margolis R, Gentry WD. Multivariate analyses of the MMPI profiles of low back pain patients. J Behavioral Med 1978;1:253-72.

10. Keefe FJ, Bradley LA, Crisson JE. Behavioral assessment of low back pain: identification of pain behavior subgroups. Pain 1990;40:153-60.

11. Williams DA, Keefe FJ. Pain beliefs and the use of cognitivebehavioral coping strategies. Pain 1991;46:185-90.

12. Williams DA, Urban B, Keefe FJ, Shutty MS, France R. Cluster analyses of pain patients' responses to the SCL-90R. Pain 1995; 61:81-91.

13. Turk DC, Okifuji A, Sinclair JD, Starz TW. Differential responses by psychosocial subgroups of fibromyalgia syndrome patients to an interdisciplinary treatment. Arthritis Care Res 1998;11:397-404.

14. Wolfe F. The relation between tender points and fibromyalgia symptom variables: evidence that fibromyalgia is not a discrete disorder in the clinic. Ann Rheum Dis 1997;56:268-71.

15. Petzke F, Gracely RH, Park KM, Ambrose K, Clauw DJ. What do tender points measure? Influence of distress on 4 measures of tenderness. J Rheumatol 2003;30:567-74.

16. Radloff LS. The CES-D scale: a self-report depression scale for research in the general population. Applied Psychological Measurement 1977;1:385-401.

17. Hertzog C, Van Alstine J, Usala PD, Hultsch D, Dixon R. Measurement properties of the Center for Epidemiological Studies Depression Scale (CES-D) in older populations. Psychological Assessment 1990;2:64-72.

18. Preliminary manual for the State-Trait Personality Inventory (STPI). Spielberger CD, editor. Palo Alto: Consulting Psychologists Press; 2000.

19. Manual for the State-Trait Anxiety Inventory (STAI) (self-evalu- ation questionnaire). Spielberger CD, Gorsuch RL, Lushene R, editors. Palo Alto: Consulting Psychologists Press; 1979.

20. Rosenstiel AK, Keefe FJ. The use of coping strategies in chronic low back pain patients: relationship to patient characteristics and current adjustment. Pain 1983;17:33-44.

21. Ware JEJ, Sherbourne CD. The MOS 36-item short-form health survey (SF-36). I. Conceptual framework and item selection. Medical Care 1992;30:473-83.

22. Beaton DE, Hogg-Johnson S, Bombardier C. Evaluating changes in health status: reliability and responsiveness of five generic health status measures in workers with musculoskeletal disorders. J Clin Epidemiol 1997;50:79-93.

23. Petzke F, Khine A, Williams D, Groner K, Clauw DJ, Gracely RH. Dolorimetry performed at 3 paired tender points highly predicts overall tenderness. J Rheumatol 2001;28:2568-9.

24. Gracely RH, Dubner R, McGrath PA. Narcotic analgesia: fentanyl reduces the intensity but not the unpleasantness of painful tooth pulp sensations. Science 1979;203:1261-3.

25. Gracely RH, Lota L, Walter DJ, Dubner R. A multiple random staircase method of psychophysical pain assessment. Pain 1988;32: 55-63.

26. Milligan GW. A review of Monte-Carlo tests of cluster-analysis. Multivariate Behavioral Research 1981;16:379-407.

27. Buckelew SP, Parker JC, Keefe FJ, Deuser WE, Crews TM, Conway R, et al. Self-efficacy and pain behavior among subjects with fibromyalgia. Pain 1994;59:377-84.

28. Dickens C, Jayson M, Sutton C, Creed F. The relationship between pain and depression in a trial using paroxetine in sufferers of chronic low back pain. Psychosomatics 2000;41:490-9.

29. White KP, Nielson WR, Harth M, Ostbye T, Speechley M. Chronic widespread musculoskeletal pain with or without fibromyalgia: psychological distress in a representative community adult sample. J Rheumatol 2002;29:588-94.

30. Croft P, Schollum J, Silman A. Population study of tender point counts and pain as evidence of fibromyalgia. BMJ 1994;309:696-9. 